\title{
Non-invasive Estimation of Stress in Conflict Resolution Environments
}

\author{
Paulo Novais, Davide Carneiro, Marco Gomes, José Neves

\section{DI-CCTC} \\ University of Minho \\ Largo do Paço, 4704-553 Braga, Portugal \\ \{pjon; dcarneiro\}@di.uminho.pt,.pg18373@alunos.uminho.pt, jneves@di.uminho.pt
}

\begin{abstract}
The current trend in Online Dispute Resolution focuses mostly on the development of technological tools that allow parties to solve conflicts through telecommunication means. However, this tendency leaves aside key issues, namely our concern with respect to context information that was previously available in traditional Alternative Dispute Resolution processes. The main weakness of this approach is that conflict resolution may become a cold process, focused solely on objective questions. In order to overcome this inconvenience, we move forward to incorporate context information in an Online Dispute Resolution platform. In particular, we consider the estimation of the level of stress of the users by analyzing their interaction patterns. As a result, the conflict resolution platform or the mediator may weight to what extent a party is affected by a particular matter, allowing one to adapt the conflict resolution strategy to a specific problem in real time.
\end{abstract}

Keywords: Multi-agent systems, Online Dispute Resolution, Stress, Influence Diagram Model.

\section{Introduction}

Online Dispute Resolution is now seen as the new technology-based paradigm for solving disagreements, and then replacing litigation in court. However, as the human's role gradually loses its substance as the main decision maker, some elements must be taken into consideration, so that conflict resolution processes guided by autonomous software agents will incorporate the best facets of the human experts [1]. Concerning interpersonal communication, Mehrabian [2] points out that the non-verbal elements are particularly important for communicating feelings and attitudes. In that sense, the use of technological tools for communication, with the consequent separation of the interlocutors may represent a risk, as a significant amount of context information is lost.

It is our conviction that these issues should be taken into consideration when developing technology-based conflict resolution platforms. Specifically, we believe that the most suited approach merges insights from Multi-Agent Systems (MAS) [11] and Ambient Intelligence (AmI) [3, 10], which we have applied previously with success in other domains $[12,13]$.

Our objective is to use intelligent environments to support the conflict resolution process. Basically, we are extending the traditional technology-based conflict resolution model, in which a user simply interacts with the system through 
a simple interface, with a new component, an intelligent environment.

From all the different sources of context information that we could consider, we are currently working on the estimation of the levels of stress. Stress has spawned a vast body of research in both the health and occupational literature [5]. In fact, some research areas on the topic of stress can be identified, namely [6]: (1) stressors (the environment causes of stress), (2) intervening variables and (3) strains (the outcomes of stress).

The agent-based approach followed in this work makes the developed system a highly modular one, easily adaptable to other domains. In this paper, we focus in the development of two novel types of agent, able to estimate the level of stress of human users in a non-intrusive way.

Our goal is to develop a dynamic conflict resolution model that, while making use of this context information, will adapt strategies in order to shape the models used by human experts. In fact, human mediators frequently make changes in their strategies when they detect significant changes in the state of the parties [4]. With this approach we expect to see the advent of environments whose main objective is to capture context information that can be used by conflict resolution platforms to achieve better and more satisfactory outcomes for the involved parties.

\section{UMCourt}

UMCourt is an agent-based conflict resolution platform being developed under the TIARAC project. This is a project funded by the Portuguese government whose main objective is to look at how Artificial Intelligence techniques can be used to improve the legal domain. The core of the platform is a group of information retrieval algorithms that are able to transparently support a wide range of high level services such as searching for past similar cases, computing the likeliness of a solution or compiling useful information, just to name a few. Figure 1 shows the organization of the agents of the UMCourt platform. We will not describe them in detail as this has already been subject of previous publications [14]. We will rather focus, for the rest of the paper, in the description of the two new agents that are extending this platform.

\subsection{Extending UMCourt}

In order to extend this architecture with a first feature of determining the level of stress, two new types of agents were created:

- Stress Manager - is responsible for receiving information from Stress Sensors and estimate a value of stress for a given user;

- Stress Sensor - multiple instances of this agent exist in the architecture, one for each different source of information about stress. Stress Sensors register with a Stress Manager.

The Stress Manager is responsible for receiving information from the Stress 
Sensors and computing an estimation of the level of stress. Currently, four different stress sensors have been developed and are being tested: Personal Conflict Style, Touch/Accuracy, Accelerometer and Movement.

The Personal Conflict Style deduces stress information from the interaction of the parties, by analyzing the utility of the proposals they create and how they react to each proposal they receive (e.g. accept, reject, reply). In a few words, specific conflict resolution styles are associated to different levels of stress (e.g. a collaborative party is usually more relaxed than a competitive one).

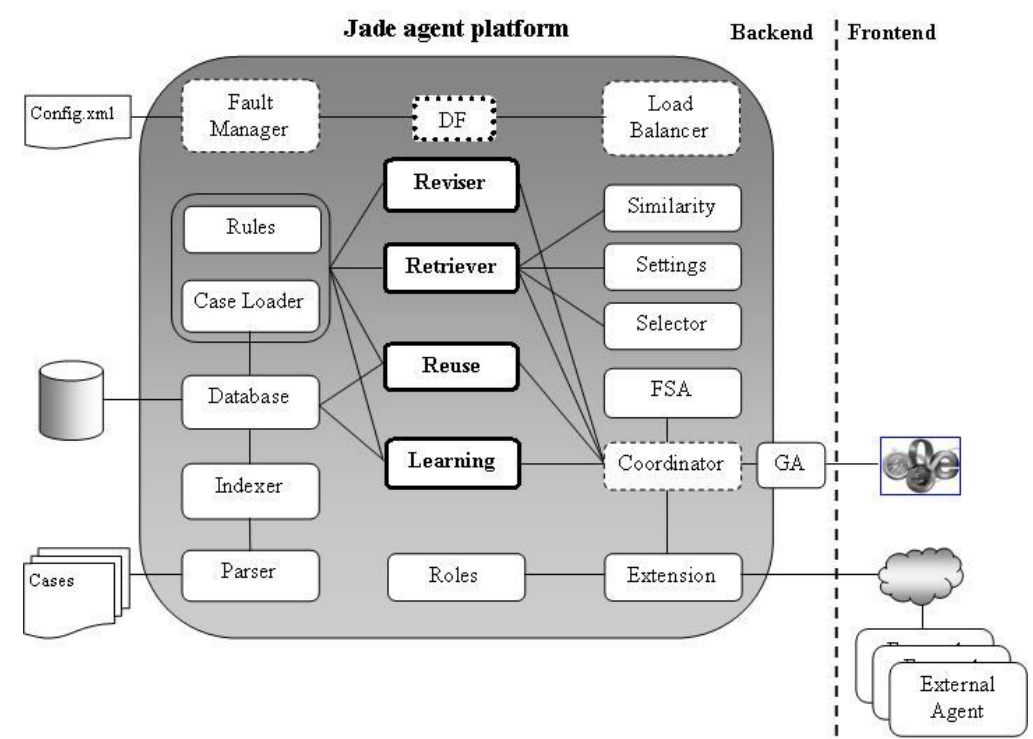

Fig. 1. The organization of the agents that make up the UMCourt architecture.

The second stress sensor concerns the touch patterns of the user while interacting with the interface. Specifically, we are interested in analyzing the intensity and the accuracy of the touch. This is the sensor that revealed the most interesting results so it will be detailed further ahead.

We also consider information about the accelerometer of the mobile interface under the assumption that higher levels of stress reflect on the way that the user carries and uses his handheld device. Finally, the last stress sensor we are currently working on concerns a video camera and involves measuring the amount of movement of the user, basically by analyzing the amount of pixels that change from one frame to the other. The agent's organization is dynamic so that the Stress Manager can compute a value of stress even when some of the sensors are not available. The agent-oriented nature of this approach also makes it easier to adapt this functionality to other domains.

\section{A Dynamic Influence Diagram Model for Stress Recognition}


Howard and Matheson introduced the Influence Diagrams (IDs) in 1981 [8]. Since then, IDs have been widely used as a knowledge representation framework to facilitate decision making and probabilistic inference. An ID is a directed acyclic graph consisting of nodes and the directed links. Nodes are grouped into decision nodes, chance or random nodes, and utility nodes, while directed links characterize probabilistic relationships or time precedence between nodes.

In the Decision-Theoretic Framework for Affect Recognition and User Assistance, presented by Liao et al. [7], the IDs are used to capture diverse sources of knowledge in decision making for stress recognition, namely: (1) conditional relationships about how events influence one another in decision domain, (2) informational relationship about what action sequences are feasible in a given set of circumstances, and (3) functional relationships about how desirable the consequences are [9]. The authors present a dynamic ID for modeling the temporal evolution of stress. In general, a dynamic ID is made up of interconnected time slices of static IDs, and the relationships between two neighboring time slices are modeled by a Hidden Markov Model, i.e., random variables at time $\mathrm{t}$ are affected by variables at time $\mathrm{t}$, as well as by the corresponding random variables at time t-1. The diagram depicted in Figure 2 is based in the Dynamic Influence Diagram Model cited above.

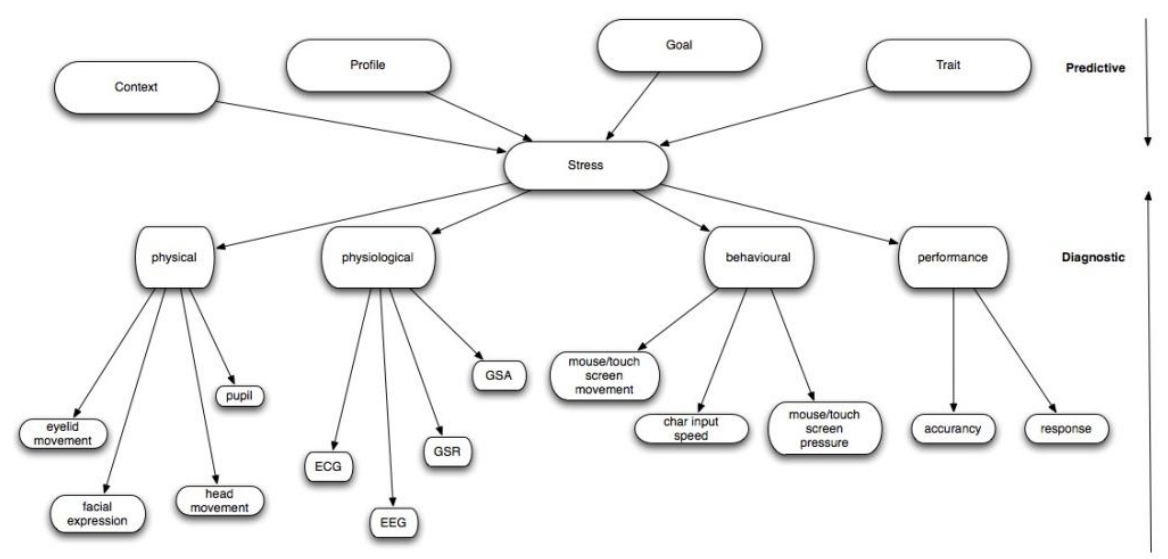

Fig. 2. A generic dynamic influence Diagram model for recognizing human stress.

This is a generic diagram that models the features and the causes that are related to user stress recognition. The upper part of the diagram, from the top to the stress node, depicts some of the elements that can alter human stress. This is considered as the predictive part and models the contextual factors that can alter/cause user stress. The lower part of the diagram, from the stress node to the leaf nodes, depicts the observable features that reveal stress. This part is called diagnostic. These features include quantifiable measures on the user's physical appearance, physiology, behaviors or performance. This generic model is flexible enough to allow variables to be inserted and modified. For example, the random 
variables under the physiological node may change, depending on the availability of required measuring devices.

\section{Results and Conclusions}

Figure 3 shows an example of the evolution of the stress for a given timeframe. The line of stress evolution shows how the stress evolved until $t=10$, considering the increased weight of more recent values. This information is received by the conflict resolution platform that may, in turn, inform the mediator. Based on this information, the (electronic or human) mediator is able to perceive how the state of each party is evolving. Moreover, the mediator is able to see if, after proposing a given solution, the level of stress of each party increases or decreases and take future decisions based on that analysis.

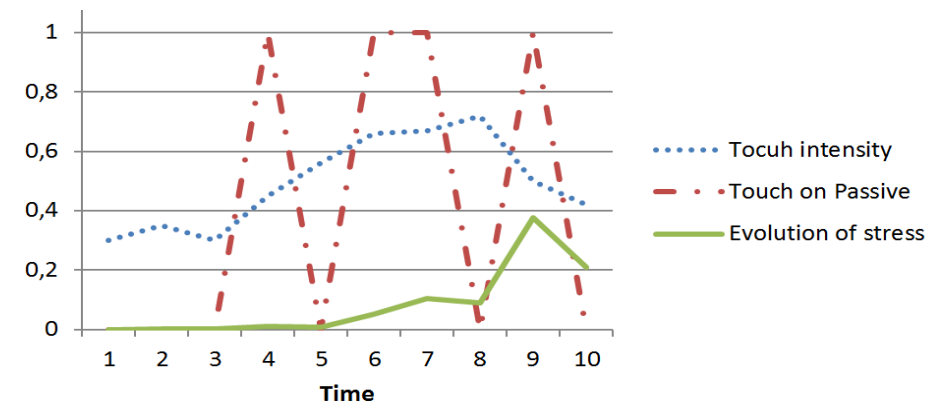

Fig. 3. Example of the evolution of the user stress according to the touch intensity and the number of touches on passive areas.

Current trends on Online Dispute Resolution are focusing on developing tools that can help parties to get into contact and share information and proposals for problem resolution. This is expected to result in faster and more efficient conflict resolution processes. However, the humanistic side of the conflict resolution is being left aside, as pointed out by the literature. Consequently, we must have in mind that there is the risk of excluding important context information that was once considered by expert human mediators to take their decisions. As a result, conflict resolution processes can get cold and focused only on the objective information, setting aside context information that may be quite important, mainly from the mediator's perspective.

The approach presented has as main objective to enrich conflict resolution platforms with access to this context information. Specifically, in this paper we focused on how to estimate the level of stress from the users. This information can then be used by either the platform or even a mediator that is conducting the process, to perceive how each issue or event is affecting each party. This, we believe, will increase the rate of success of conflict resolution procedures and bring them closer to the rich communicative environment that we have, when we 
communicate face-to-face.

Acknowledgments. The work described in this paper is included in TIARAC Telematics and Artificial Intelligence in Alternative Conflict Resolution Project (PTDC/JUR/71354/2006), which is a research project supported by FCT (Science \& Technology Foundation), Portugal. The work of Davide Carneiro is also supported by a doctoral grant by FCT (SFRH/BD/64890/2009).

\section{References}

1. Larson, D.: Technology Mediated Dispute Resolution. Proceeding of the 2007 conference on Legal Knowledge and Information Systems: JURIX 2007: The Twentieth Annual Conference. IOS Press Amsterdam, The Netherlands (2007)

2. Mehrabian, A.: Silent Messages - A Wealth of Information about Nonverbal Communication. Personality \& Emotion Tests \& Software. Los Angeles (2009)

3. Aarts E., Grotenhuis F., Ambient Intelligence 2.0: Towards Synergetic Prosperity, Journal of Ambient Intelligence and Smart Environments 3, IOS Press, (2011) 3-11

4. Lewick, R., Weiss2, S., Lewin, D.: Models of Conflict, Negotiation and Third Party Intervention: A Review and Synthesis. Journal of Organizational Behavior, Volume 13, Issue 3 (1992) 209-252

5. Mulligan, E.: Approaches to measuring stress - A literature review. (2007) available at http://www.superstar.org.uk/Resume/EdMulligan/Postgrad/StressLiteratureReview.pdf <accessed September 2011>

6. Jones, F., Kinman, G.: Approaches to Studying Stress. In F. Jones \& J. Bright (Eds.), Stress: Myth, Theory and Research. Harlow: Pearson Education (2001)

7. Liao, W., Zhang, W., Zhu, Z., Ji, Q.: A Decision Theoretic Model for Stress Recognition and User Assistance, presented at the Twentieth National Conference on Artificial Intelligence (AAAI-05) (2005)

8. Howard, R.A. Matheson, J.: Readings on the Principles and Applications of Decision Analysis, Strategic Decisions Group, Menlo Park (1981)

9. Pearl, J.: Probabilistic reasoning in intelligent systems: networks of plausible inferences. Morgan Kaufman Publishers (1988)

10.Novais, P., Costa, R., Carneiro, D., Neves, J., Inter-Organization Cooperation for Ambient Assisted Living, in Journal of Ambient Intelligence and Smart Environments, IOS Press, Vol. 2, No. 2, pp.179-195, ISSN 1876-1364 (2010)

11.Wooldridge, M., Jennings, N.R., Intelligent Agents: theory and practice, In The Knowledge Engineering Review, p. 115-152 (1995)

12.Carneiro, D., Novais, P., Costa, R., Neves, J., Developing Intelligent Environments with OSGi and JADE, Artificial Intelligence in Theory and Practice III, Bramer M. (Ed), IFIP AI, Springer (2010)

13.Carneiro, D., Novais, P., Costa, R., Neves, J., Enhancing the Role of Multi-agent Systems in the Development of Intelligent Environments, In Trends in Practical Applications of Agents and Multiagent Systems (PAAMS), Advances in Intelligent and Soft Computing, Springer (2010)

14.Carneiro, D., Novais, P., Neves, J., An Agent-based Architecture for Multifaceted Online Dispute Resolution Tools, in Developing Concepts in Applied Intelligence, Mehrotra K., Mohan C., Oh J., Varshney P., Ali M. (eds), Springer - Studies in Computational Intelligence, ISBN: 978-3-642-21331-1, pp. 89-94 (2011) 